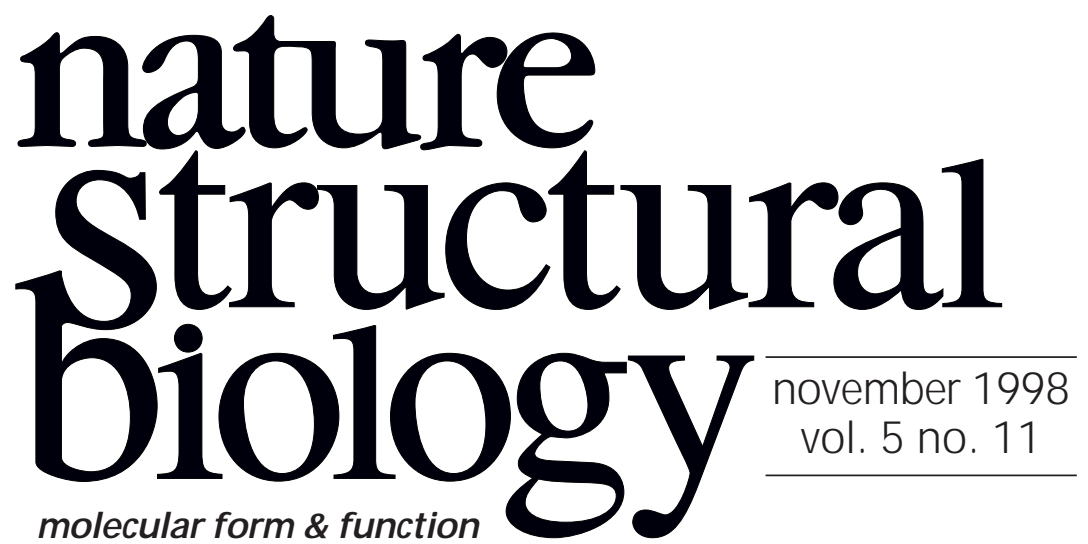

\title{
The 'new' PDB
}

On October 1, 1998, the Research Collaboratory for Structural Bioinformatics (RCSB) ${ }^{1}$ was officially awarded a grant to assume operation of the Protein Data Bank (PDB) ${ }^{2}$ and will replace Brookhaven National Laboratory (BNL) as caretaker over the coming year. News of the decision to move the PDB began to spread through the scientific community during the last week of August, well before the final funding agreement had been signed. The lack of concrete information about the plans for the Macromolecular Structure Database (MSD), as the 'new' PDB may be called, has led to a feeling of concern among structural biologists ${ }^{3}$. Now, scientists may assess the merits of the two proposals for themselves - both are available online $e^{4,5}$.

History. The PDB was established in 1971 at BNL by an international group of scientists and was supported by the National Science Foundation (NSF) from 1971-1991. In 1992, responding to a rise in the number of deposited structures, additional support was obtained from the Department of Energy (DOE), the National Institutes of Health (NIH) and the National Library of Medicine (NLM). Throughout the first 20 years, there were no funding competitions; the grant review program for the operation of the PDB began only in 1994. BNL was awarded the first grant from this process. Since 1995, the PDB, under the continuing supervision of Joel Sussman, has been funded by a cooperative agreement providing $\sim 9$ million US dollars over four years. Earlier this year, another competition was initiated by the NSF with the following guidelines ${ }^{6}$ : "proposals ... must discuss the structure of the proposed database, including the format of data entries, and provide detailed plans for long-term management and distribution of the database. [Additonally], the data should be structured and maintained in a way that permits the development and use of complex queries by knowledgeable users, including commercial software developers". How did each group offer to meet these goals?

The two proposals. The three senior investigators of the PDB - Joel Sussman (Head of the PDB) Enrique Abola (Deputy Head) and Otto Ritter (Head of Informatics) — focused most of their attention on the need to improve deposition processes and to maintain reliability of the archive ${ }^{4}$, an admittedly enormous task given current (about four structures deposited per day) and estimated future rates of deposition. Today, the $\mathrm{PDB}^{2}$ exists as a set of 8,338 separate 'flat' files that have been deposited by researchers and reviewed by PDB staff, with final release of a completed file occurring usually $\sim 100$ days after deposition. Over the past few years, Sussman's group has performed some admirable tasks: eliminating a large deposition backlog; introducing the web based AutoDep process and the 3DB Browser and PDB Lite simple search engines; collaborating with the European Bioinformatics Institute in their handling of many European data depositions and in data harvesting, whereby data refinement programs are written to produce a file that can be easily transmitted to the PDB; and responding to the need for quick release of coordinates by developing the Layered release system $^{2}$. The PDB staff had shown that they could maintain the archive and effectively distribute the data around the world through its system of mirror sites, and they were eager to continue their work. Their proposal focused almost entirely on the archive, describing how they would move toward increased automation and efficiency of data handling and distribution. Although they do briefly mention future implementation of 3DBase, a relational database that would allow more sophisticated query searches, the overriding strong focus was on basic archiving practices, and this may have hindered acceptance of their proposal.

The RCSB proposal ${ }^{5}$, in contrast, focused much attention not just on basic archive maintenance, but also on many key problems of implementing a state of the art database, including: improving 


\section{Coordinate updates for two recent structural models published in Nature Structural Biology:}

Hanein, D., Volkmann, N., Goldsmith, S., Michon, A.M., Lehman, W., Craig R., DeRosier, D., Almo, S. and Matsudaira, P. An atomic model of fimbrin binding to F-actin and its implications for filament crosslinking and regulation. Nature Struct. Biol. 5, 787-792 (1998)

Coordinates for the model and related material are available at

http://www.bio.brandeis.edu/pages/ faculty/Derosier.html

Poirier, M.A., Xiao, W., Macosko, J.C. Chan, C., Shin, Y.-K. and Bennett, M.K. The synaptic SNARE complex is a parallel four-stranded helical bundle. Nature Struct. Biol. 5, 765-769 (1998).

The PDB accession number for the model of the SNARE complex, based on electron paramagnetic resonance spectroscopy data, is $2 \mathrm{BU} 0$.

1. Research collaboratory for structural bioinformatics. http://www.rcsb.org

2. Protein data bank. http://www.pdb.bnl.gov

3. Marshall, E. Science 281, 1584-1585 (1998).

4. Sussman, J.L. et al. The protein data bank proposal for the macromolecular structure database. http://www.pdb.bnl.gov/pdbdocs/proposal.htm

5. Berman, H. et al. RCSB's grant proposal. http://www.rcsb.org/pdb/docs/grant/toc.htm

6. National science foundtion directorate for biological sciences macromolecular structure database proposal solicitation. http://www.nsf.gov/pubs/1998/nsf9866/nf 9866htm

7. DNA binding protein structures database http://ndb.sdsc.edu/NDB/structurefinder/dnabind/

8. Nucleic acids database. http://ndbserver.rutgers.edu/

9. Biological macromolecule crystallization database and the NASA archive for protein crystal growth data (version 2.00). http:/h178133.nist.gov:4400/bmcd/bmcd.htm

10. The protein kinase resource. http://www.sdsc.edu/kinases/

11. Archive of obsolete PDB entries http://pdbobs.sdsc.edu/

12. The RCSB's protein data bank (PDB) frequently asked questions http://www.rcsb.org/pdb/docs/FAQ.html data quality; establishing file formats and annotating all PDB files to allow complex queries; and developing an architecture that will allow easy incorporation of other databases (for example, ones developed by scientists in specific fields, such as serine proteases) into the search platform. The RCSB is a consortium composed of Rutgers, the State University of New Jersey; the San Diego Supercomputer Center (SDSC) of the University of California at San Diego (UCSD); and the National Institute of Standards and Technology (NIST). According to Helen Berman, the principal investigator of the RCSB MSD, there are two major problems with the current PDB system that they intend to remedy: (i) the deposition procedure, although much better than it used to be, is inflexible and processing of data files is cumbersome, taking months to complete; and (ii) the files are 'flat' there is no formal data representation as used in modern relational database technologies - and thus searches and queries are limited to what can be easily extracted from the separate, inconsistently annotated files that have been collected in different formats over the years. To allow complex searches, different groups have been required to download particular files from the PDB archive, to complete missing information and to place the data into a searchable table format - a process known as curation. For example, the database of DNA-binding protein structures ${ }^{7}$ was assembled in this way.

The members of the RCSB have experience in building and maintaining databases (for a sample of their efforts, see refs 8-11). Each group, according to their different strengths, will have defined responsibilities in the MSD: Rutgers will handle data deposition, validation and annotation; NIST will oversee data uniformity, evaluation and master archiving; and the SDSC/UCSD group will manage data integration and distribution. Although having a separated management team will be a new model, over the past six months these groups have worked together closely to demonstrate that their vision of a reliable, searchable modern database can be accomplished. For example, they have already curated $\sim 2,000$ of the $\sim 8,000 \mathrm{PDB}$ data files, to prepare them for use in the MSD system. They estimate that the entire current PDB content will be fully annotated and searchable in detail within two years, and are proceeding with curation by protein family (for example, work on the globins is ongoing).

A common worry expressed by the community is that the RCSB may be promoting flashy technology at the expense of reliable deposition and archiving practices. However, the RCSB groups believe that they can provide, in addition to increased query capability, more reliable and useful deposition, archival and integration processes than those used currently by the PDB. In particular, they expect to reduce the average time of file review to about two weeks (as is now standard practice with the Nucleic Acids Database ${ }^{8}$, operated by Rutgers). They also plan to improve validation procedures. For example, the RCSB is initiating a task force to determine what types of validation, specific to NMR data, should be included in standard software. Integration with other databases should also be a key feature of the new MSD; in their proposal, many cross links, such as to Medline and the mouse gene database, are listed as 'in progress'. To achieve their integration goals, the RCSB is developing software for automated searching of remote sites and updating of files. The RCSB vision of the new MSD is rich, and if accomplished, should provide a useful tool for biologists.

Transition. The response from many scientists has been: why change? The BNL PDB group has had good standing within the community and since the PDB is one of the world's most important databases, many researchers would be happier with continuity. However, the accomplishments of the RCSB, separately over the years and together over the past few months in developing plans for the MSD, indicate that they are up to the task of operating and even improving this valuable resource. While individuals, after evaluating the two proposals $s^{4,5}$, may agree or disagree, the decision has been made. How will the transition occur? Both Berman and Sussman have stated emphatically that the transition will be smooth and transparent - both groups will work together closely to insure rapid and efficient transfer of necessary tools and information. Originally, the RCSB had planned to assume all of its responsibilities on Day One of an award, as they state in their proposal 5 . However, Berman has said that, while they are capable of doing so, they have realized that there is and will continue to be much concern in the community about rushing the transfer. In response, the RCSB has posted a list of frequently asked questions and answers on their web site ${ }^{12}$. Berman notes that they are keen to avoid confusion. Therefore, the RCSB will gradually phase in tools and take over responsibility from the PDB. For now, both depositors and users should continue to use the BNL PDB site ${ }^{2}$. In the near future, a time table of proposed changes will be posted on the web sites and changes will be noted clearly as they occur. Additionally, prior to release of new software, the RCSB will conduct tests, utilizing volunteers representing all types of MSD users. Furthermore, at the request of the NSF, the RCSB is preparing a detailed transition plan to be reviewed by an advisory board as well as by the NSF.

The future. In 1973, 10 structures were deposited into the PDB; in 1983, 27; in 1993, 922 (ref. 2). What will the deposition number be in 2,003, as the five year, 10 million US dollar initial award to the RCSB nears its end and another competition is initiated? One can only guess. Clearly, biologists will need a reliable and scalable database to accept, annotate and interpret structural information. The RCSB's MSD looks good on paper. In the face of such growth, everyone hopes that it can deliver. 